\title{
Eduardo Menéndez. Doctorado Honoris Causa por la Universidad Rovira i Virgili
}

\author{
Eduardo Menéndez. Honorary Doctorate \\ by the Rovira i Virgili University
}

El jueves 21 de mayo de 2009, Eduardo Menéndez, investigador del Centro de Investigaciones y Estudios Superiores en Antropología Social (CIESAS) de México, e integrante del Consejo Editorial de nuestra revista, fue investido con el título de Doctor Honoris Causa por la Universidad Rovira i Virgili (URV) de Tarragona (Cataluña, España), tal como lo fueron Amartya Sen y Noam Chomsky, entre otros. La postulación, propuesta por el Departamento de Antropología, Filosofía y Trabajo Social de la URV, es un merecido reconocimiento público a su trayectoria profesional y a la relevancia de su obra.

El texto que presentamos a continuación, contiene algunos fragmentos desgrabados de la exposición a cargo de Joseph Comelles y de la conferencia pronunciada por Eduardo.

En el marco de una ceremonia engalanada con togas negras, birretes y capas, la lectura del laudatium estuvo a cargo del padrino del doctorando, el Dr. Joseph Comelles, quien describió su primer acercamiento a la obra de Eduardo:

\begin{abstract}
"Hace casi 30 años, la profesora Dolores Juliano, [...] exiliada en Barcelona ante las amenazas del régimen militar de la República Argentina, nos mandó un manuscrito para publicar en nuestra revista Arxiu d'etnografia de Catalunya. Era de un compañero suyo, Eduardo Menéndez, exiliado como ella, en México. Cincuenta páginas a doble espacio, máquina de escribir "Atlas" de aquellas de las antiguas, anotaciones en los márgenes con estilográfica. Título: El modelo hegemónico: transacciones y alternativas hacia una fundamentación teórica del modelo de autoatención en salud. La lectura fue una bocanada de aire fresco, esclarecedora de ideas y cuestiones que desde aquí habíamos sido incapaces de resolver. Emergía el papel de la medicina hegemónica en el contexto de un pluralismo asistencial. Impresionados lo publicamos".
\end{abstract}

Destacó además su papel docente, como maestro de varias generaciones de discípulos en España y América Latina, y en Italia:

"...discípulos al viejo estilo, como los que viajaban en la Edad Media a Montpellier para escuchar a Arnaldo de Vilanova o a la Sorbona a Tomás de Aquino. Una relación maestro discípulo basada

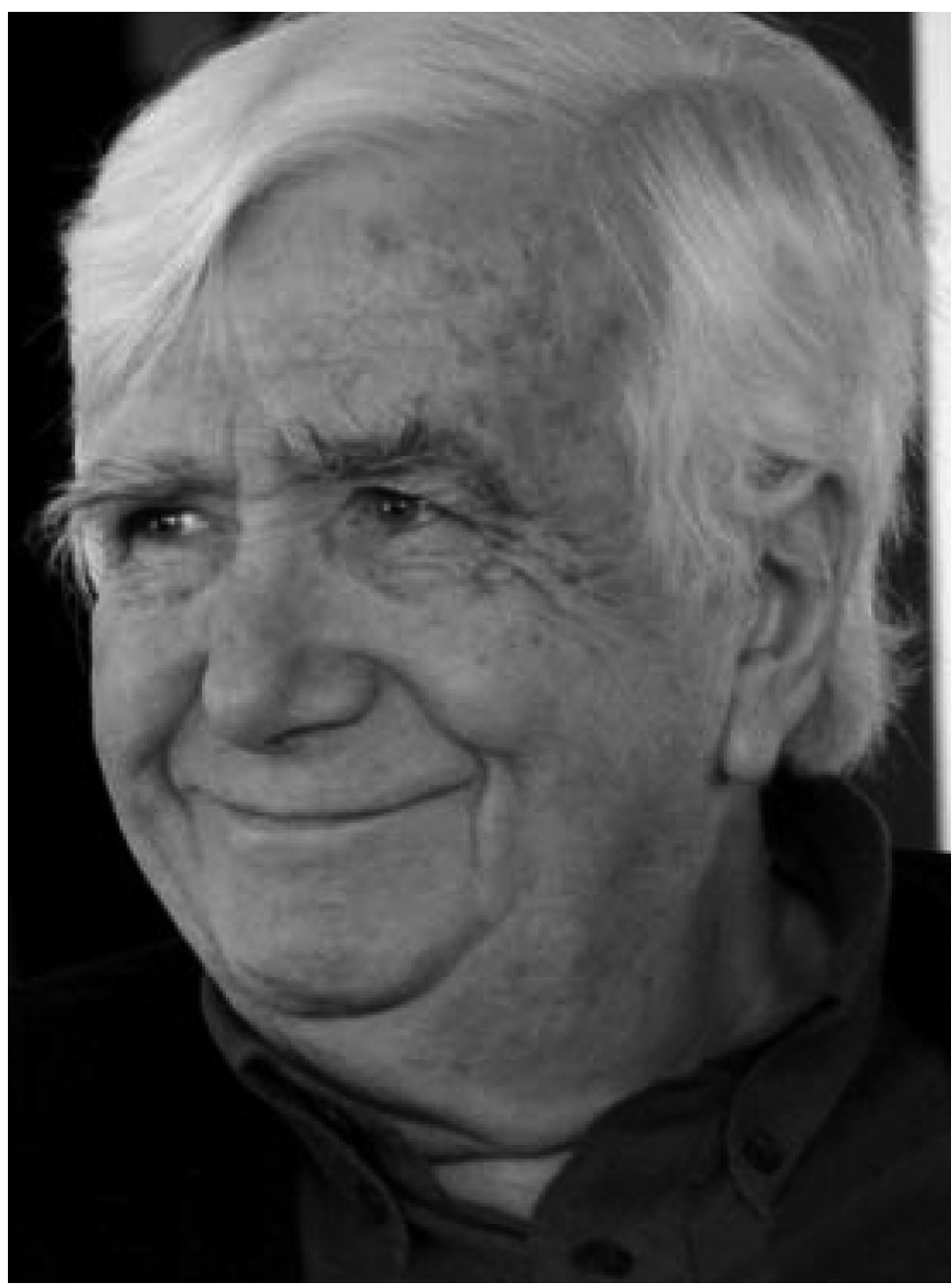

en contactos personales, en clases, en documentos publicados en ediciones de 300 ejemplares por instituciones de nombres misteriosos, que viajaban en equipajes de mano en los aviones y que una vez aquí o allá se fotocopiaban una y 
otra vez, una y otra vez, para que ese saber circulase de mano en mano, de boca en boca. Fotocopias que a menudo venían con anotaciones de varias manos, único remedio de un universo, casi al estilo de los copistas medioevales, en el que el índice de impacto no tenía ningún valor, y en el que el alimento que significa la producción teórica y la discusión universitaria circula como cuando en Bolonia se fundó la primera universidad cara a cara, en clases a menudo en una terraza de café con unas sobremesas y lejos de la parafernalia supuestamente pedagógica de la versión carpetovetónica de la actual Bolonia".

Luego del ritual de investidura, el Dr. Eduardo Menéndez, con su birrete distintivo, saludó y agradeció a los presentes:

"...estoy más conmovido de lo que yo hubiera pensado, no solamente por la ceremonia en sí, sino por las palabras de Joseph. [...] Agradezco profundamente al Consejo de Gobierno y al Rector de la Universitat Rovira i Virgili, el otorgamiento del Doctorado Honoris Causa y dicho agradecimiento no es solo por el doctorado en sí, sino por lo que representa para mí tanto en términos académicos y profesionales como también en términos afectivos y de amistad, dado que este título constituye una especie de símbolo objetivo del trabajo que realizamos a lo largo de casi 25 años con los colegas y amigos del departamento de antropología de esta universidad y entre los que destaco especialmente a los doctores Joseph María Comelles y Oriol Romaní, así como también a las sucesivas generaciones de alumnos a los que acompañamos en el proceso de formarse como antropólogos médicos. Y es por eso además que agradezco la presencia de todos ustedes, de los colegas y amigos que han venido desde México y otros lugares de España y especialmente de mi esposa y de mis dos hijas con las cuales comparto este reconocimiento".

En su conferencia rescató los aportes de los diversos autores que influyeron de manera fundamental en su propia reflexión teórica y en la práctica de su quehacer antropológico, como Émile Durkheim, Claude Lévi-Strauss, entre otros:
"Considero que Durkheim no solo es uno de los fundadores de lo que denominamos enfoque relacional aplicado al estudio de los procesos de salud-enfermedad-atención, sino que este autor nos propone tempranamente que los padecimientos no pueden reducirse al sujeto ni aún tratándose del suicidio, sino que dicho sujeto y su padecimiento deben ser referidos a la red de relaciones dentro de las cuales se enferma y soluciona -o no soluciona- su padecimiento. Y será en el desarrollo de sus análisis sobre el suicidio que Durkheim propondrá lo que hoy conocemos con el nombre de redes sociales y grupo sostén, lo cual cuando los propuso no fueron realmente muy bien entendidos y pasaron muchos años antes de que fueran utilizados no solamente en términos técnicos, sino sobre todo de intervención".

Al finalizar, retomando parte del desarroIlo de su conferencia, concluyó con las siguientes palabras:

"Como señalé al principio por lo menos una parte del trabajo antropológico es autobiográfico. Yo tardé varios años en darme cuenta de que el primer trabajo más o menos serio que yo realicé, y me refiero al estudio sobre la migración de población italiana y española a una mediana comunidad de la provincia de Entre Ríos, en Argentina, tenía que ver no solo con la problemática teórica y etnográfica de las migraciones, sino conmigo, como un sujeto hijo y nieto de inmigrantes españoles e italianos que se radicaron en la Argentina entre 1910 y 1920. Y es a partir de este último señalamiento que considero que el principal aporte de la antropología actual ha sido el de focalizar el estudio de lo obvio, de lo que está tan cerca que no lo vemos, de externar nuestra interioridad para poder observarla desde dentro $y$ desde afuera y en consecuencia hacer surgir obviedades. Y no cabe duda de que en esta ceremonia, es obvio, por lo menos para mí, que lo importante no es mi conferencia, sino el sentirme reconocido especialmente por mis colegas y amigos de Tarragona y de otras partes, pero no solo en términos académicos, sino especialmente como un hombre más o menos bueno, como diría Antonio Machado". 